\title{
Biological Immunotherapy
}

National Cancer Institute

\section{Source}

National Cancer Institute. Biological Immunotherapy. NCI Thesaurus. Code C15972.

The use of biological agents to enhance the host immune system. 